\title{
LASER NOISE CONSIDERATIONS FOR PHASE MODULATED LINKS
}

\author{
Joseph M. Singley ${ }^{1}$, John F. Diehl', and Vincent J. Urick ${ }^{2}$ \\ ${ }^{1}$ Sotera Defense Solutions, Inc., Crofton, MD 21114 \\ ${ }^{2}$ U.S. Naval Research Laboratory, Washington, DC 20375
}

Photonic links employing intensity modulation with direct detection are typically unaffected by laser phase noise without a mechanism to convert that phase noise into intensity noise. Such mechanisms in long links include chromatic dispersion and double-Rayleigh scattering, the latter of which introduces multi-path interference (MPI). However, these processes are generally not a concern in short links for avionic platforms except when reflections from fiber optic connectors produce strong MPI. Phase modulated links provide distinct advantages over intensity modulation for digital [1] and analog [2] applications; however, they are severely impacted by laser phase noise. Here we describe considerations for a phase modulated link employing an asymmetric Mach-Zehnder interferometer (MZI).
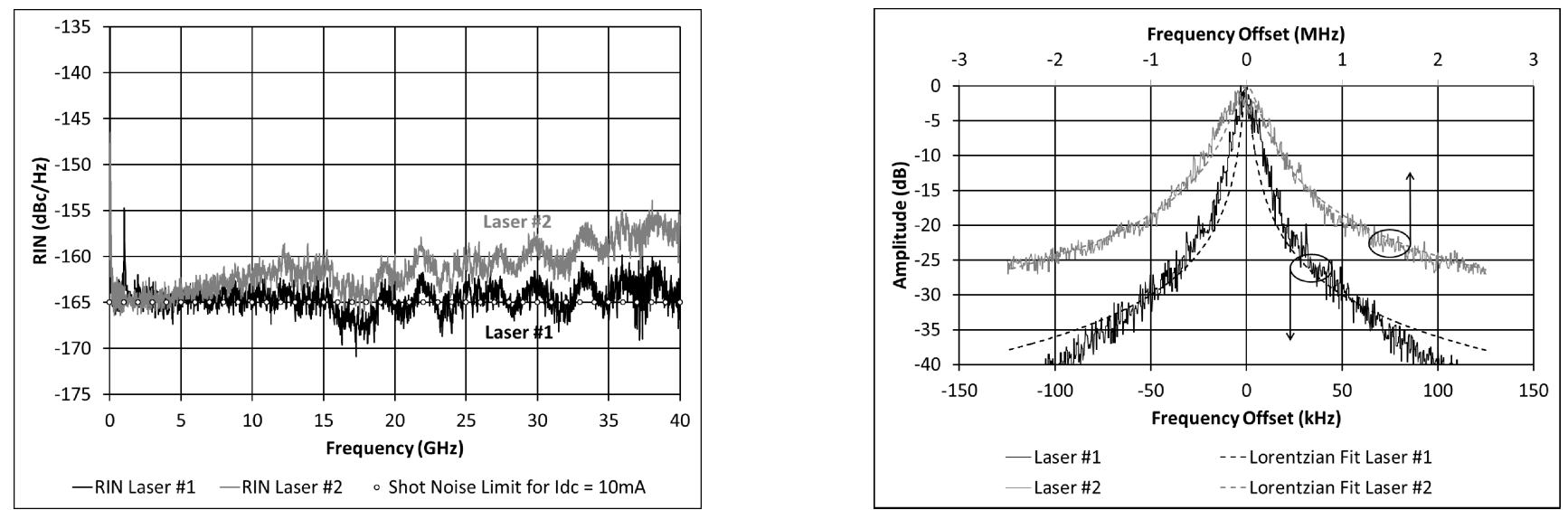

Figure 1. (a) RIN to $40 \mathrm{GHz}$ (b) Delayed Self-Heterodyne results for JDSU and Orbits Lightwave Lasers

Laser intensity noise, typically expressed as relative intensity noise (RIN), is not a sufficient enough measure by itself when selecting a laser for a phase modulated link. Figure 1a displays the RIN spectrum of two lasers. Note that the structure in the spectrum from $20-40 \mathrm{GHz}$ is due to an impedance mismatch in the system. Laser \#1 is an Orbits Lightwave Ethernal $1550 \mathrm{~nm}$ fiber laser while Laser \#2 is a CQF935/708 Series DFB laser from JDS Uniphase. Laser \#1 is shot-noise limited (SNL) to $40 \mathrm{GHz}$ and Laser \#2 is only about $5 \mathrm{~dB}$ worse at higher frequencies. However, these two lasers have significantly different linewidths as evident in Figure $1 \mathrm{~b}$ which shows the beat tone produced from a delayed self-heterodyne measurement on each laser. The full-width at half-maximum (FWHM) linewidth of Laser \#2 was determined to be $\sim 120 \mathrm{kHz}$ by way of a Lorentzian line fit algorithm. Laser \#1 on the other hand exhibits a much higher degree of spectral purity than Laser \#1 and has a linewidth that was measured to be only $1.6 \mathrm{kHz}$. It should be noted that the linewidth measurement setup used has a $37.5 \mathrm{~km}$ fiber delay which limits accurate measurements (incoherent regime) to $2.5 \mathrm{kHz}$ or greater. Also, a portion of the fiber loop was outside of the isolation chamber which allows for artificially broadening of the lineshape due to acoustic and mechanical pickup.

We employed an MZI with a differential delay of 375 ps $(F S R=2.67 \mathrm{GHz})$ to measure the phase noise of both lasers by converting it to intensity noise in the coherent regime. The resultant RIN plot can be seen below in Figure 2a. Again, note that the structure in the spectrum from $20-40 \mathrm{GHz}$ is due to an impedance mismatch in the system. This plot reveals that the primary noise source from Laser \#2 is phase noise as result of its 
increased linewidth. A cursory glance at this lasers' directly-detected RIN plot could mislead someone into selecting it for a system utilizing phase modulation with interferometric demodulation (ФMID). The mismatch between the measured and calculated data for Laser \#1 in Figure 2a is due to the overestimation of linewidth from our measurement setup. Therefore, Laser \#1 must be much narrower than the measured value of $1.6 \mathrm{kHz}$. The analytical expression for laser phase noise that is converted to intensity noise through an interferometer is given as [3], [4]

$$
\operatorname{RIN}_{\Phi \mathrm{N} \rightarrow \mathrm{IN}}=\frac{\Delta v \cdot e^{-2 \pi \tau \Delta v}}{\pi\left(\Delta v^{2}+{f_{\mathrm{rf}}}^{2}\right)}[\cosh (2 \pi \tau \Delta v)-\cos (\Omega \tau)]
$$

where $\Delta v$ is the FWHM laser linewidth, $\tau$ is the time delay due to the path imbalance in one arm of the MZI, and $f_{\text {rf }}$ is the electronic frequency. Figure $2 \mathrm{~b}$ displays a plot of (1) plus the shot-noise-limited RIN given by $\mathrm{RIN}_{\text {shot }}=2 e / I_{\mathrm{dc}}$ for different laser linewidths to show the expected phase noise through an MZI. Even with laser linewidths approaching those of Nd:YAG systems $(<1 \mathrm{kHz})$, the noise level below $2 \mathrm{GHz}$ can be as much as $15 \mathrm{~dB}$ higher than the SNL. Clearly, laser linewidth plays a significant role in the selection of lasers for phase modulated links.
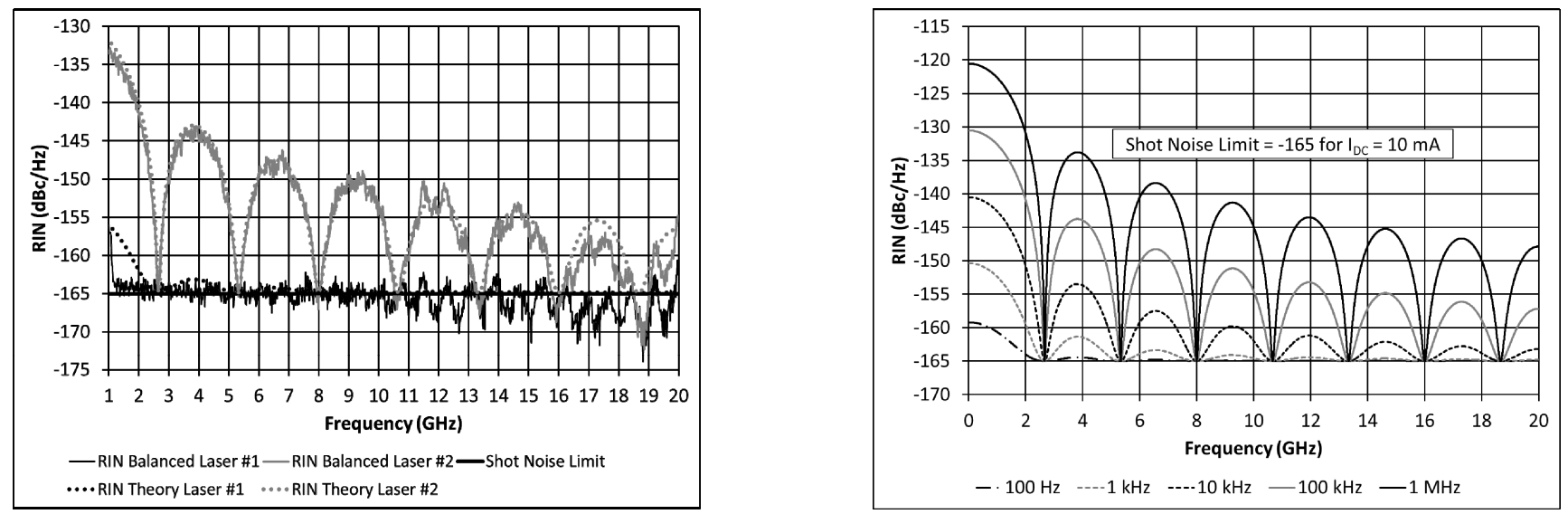

Figure 2. (a) Phase to intensity noise through MZI. (b) Calculated RIN at MZI output for various laser linewidths.

Even when lasers exhibit large directly-detected RIN peaks it is inadvisable to assume the only noise source is intensity related. While not shown here, we have observed considerable phase noise around the relaxation oscillation frequency of solid-state lasers that is not described by (1). Such emission may not be captured by a typical linewidth measurement and is detrimental to the performance of $\Phi$ MID links. In conclusion, any limitations in the performance of $\Phi$ MID links related to laser phase noise can be mitigated by careful selection of the laser by considering both its phase- and intensity-noise spectra.

\section{References}

[1] A. H. Gnauck and P. J. Winzer, "Optical phase-shift-keyed transmission,” J. Lightwave Technol., vol. 23, no. 1, pp. 115-130, Jan. 2005.

[2] V. J. Urick, et al., "Phase modulation with interferometric detection as an alternative to intensity modulation with direct detection for analog-photonic links," IEEE Trans. Microwave Theory Tech., vol. 55, no. 9, pp. 1978-1985. Sept. 2007.

[3] B. Moslehi, "Noise power spectra of optical two-beam interferometers induced by the laser phase noise," J. Lightwave Technol., vol. LT-4, no. 11, pp. 1704-1710, Nov. 1986.

[4] R. Tkach and A. Chraplyvy, "Phase noise and linewidth in an InGaAsP DFB laser," J. Lightwave Technol., vol. LT-4, no. 11, pp. 1711-1716, Nov. 1986. 